Consultant Rheumatologists (JSM \& JHG), formally trained in TAU, established Scotland's First Fast Track Referral Pathway for Suspected GCA using TAU on 21.03.17. A third Consultant $(\mathrm{SJH})$, whose formal Vascular ultrasound training occurred later, joined the Service on 10.04.18. We present here our Outcome data and also our experience of this new Service for NHS Fife, the first of it's kind in Scotland.

Methods: We prospectively recorded data on all referrals to our Fast Track Service for patients with suspected GCA from 21.03.17 onwards. NHS Fife covers a population of 370,000 and constitutes $6.8 \%$ of the Scottish Population $(5,425,000)$

Results: Up until 20.11.17 (20 months) we received a total of 142 referrals, representing an average of 7 per month, or around 2 per week. 54 $(38 \%)$ were deemed to have a very low probability of GCA based on their age, history \& CRP and were not seen or scanned. 88 (62\%) of referrals underwent Fast Track assessment including TAU (62 F; $26 \mathrm{M}$ ); Mean age 71 (range 52-88). Time from referral to assessment was as follows : (25) $28 \%$ were seen on the same day; (60) $68 \%$ were seen by the next day; (69) $78 \%$ were seen within 2 days of referral; (84) $95 \%$ were seen within 3 days of referral. 25/88 (28\%) patients were diagnosed with GCA. Of these, 14 patients had a positive TAU and did not require TAB. 9 patients had an equivocal or negative TAU and were diagnosed on TAB. Only 1/25 GCA patients experienced LOV (4\%). The patient was an 82 year old male diabetic who presented to Ophthalmology with unilateral LOV but no symptoms of GCA and also with a normal CRP $(4.1 \mathrm{mg} / \mathrm{L})$. However, he had stopped $1 \mathrm{mg}$ of prednisolone for PMR 2 weeks prior to developing LOV. TAU was positive, but in view of the atypical nature of his symptoms ("silent GCA") \& normal CRP, he also underwent $\mathrm{TAB}$, which was positive confirming the diagnosis of GCA. I patient had a negative $T A U$ and $T A B$, but was subsequently diagnosed with LVV on Axillary artery US.

Conclusion: A previous survey of 20 GCA patients diagnosed in Fife over a 2 year period (Jan 2007 - Jan 2009) recorded a 50\% rate of LOV. This was largely attributed to delayed referral. We have now successfully introduced Scotland's First Fast Track Referral Pathway for Suspected GCA using TAU. This has led to a dramatic reduction in Morbidity in terms of LOV (<5\%).

Disclosure of Interests: None declared

DOI: 10.1136/annrheumdis-2019-eular.7603

\section{AB0604 CLINICAL FEATURES AND TREATMENT IN A COHORT OF PATIENTS WITH BEHÇET DISEASE IN A TERTIARY HOPITAL OF BARCELONA}

Joan Maria Mercadé Torras, Alfredo Guillén-Del-Castillo, Segundo Bujan Rivas, Jaume Mestre Torres, Roser Solans-Laqué. Barcelona, Barcelona, Spain

Background: Behçet disease (BD) is considered a systemic vasculitis according to the Chapel-Hill classification, which occurs most frequently between latitudes 30 and $45^{\circ}$ north and in the area of the Old Silk Route. Turkey has the highest prevalence, followed by Japan. In Spain, a prevalence of 7.5 cases per 100000 persons has been estimated. The epidemiological and clinical characteristics of European patients with BD vary with respect to those of the Turkish and Japanese cohorts.

Objectives: Description of clinical features and treatment received in a cohort of patients diagnosed with $\mathrm{BD}$ in an Internal Medicine Unit of a tertiary centre from Barcelona.

Methods: Retrospective, observational study. Epidemiological, clinical and laboratory data were obtained from clinical charts. SSPS package was used to perform statistical analysis.

Results: 132 patients (56.6\% men) diagnosed over the last 30 years and followed-up until the censoring data were included. 112 (84.8\%) were Caucasians, 15 (11.4\%) from North Africa, 4 (3\%) Asiatic and 1 (0.8\%) from South America. 2 (1.5\%) patients had a family history of BD and 9 patients $(6.8 \%)$ a family history of other rheumatic diseases. $43.9 \%$ (58 patients) were HLA-B51. Oral or genital ulcers were present in 131 $(99.2 \%)$ patients and skin involvement in 106 (80\%) cases, $44.6 \%$ had erythema nodosum, $59 \%$ had acne-like lesions and $9 \%$ had cutaneous vasculitis. Ocular involvement was observed in 69 cases (52.3\%): 27 patients unilateral and 8 bilateral anterior uveitis; 14 unilateral and 3 bilateral posterior uveitis; 10 patients unilateral and 14 bilateral panuveitis; 19 unilateral retinal vasculitis and 12 bilateral. Neurological involvement was present in $37(28 \%)$ patients: 10 parenchimal disease and 17 cases nonparenchymal disease (aseptic meningitis or vasculitis); 8 patients benign intracranial hypertension, and 3 had dural sinus thrombosis. Articular involvement was recorded in 79 (59.8\%) patients (it was observed 25 monoarthritis, 24 oligoarthritis, 6 poliarthritis and 58 patients had arthralgia). Vascular involvement was present in 43 (32.6\%) cases: deep venous thrombosis in 38 patients (11 cases in locations other than the extremities); pulmonary embolism in 7; 21 thrombophlebitis; and 6 patients aneurysms (only 2 pulmonary arterial aneurysms). Digestive involvement was present in $12(9,1 \%)$ patients, with predominant colon involvement (8 cases).

The most prescribed drugs were corticosteroids (85.6\%) and colchicine (77.3\%), followed by azathioprine (36.4\%) and cyclosporine A (33.3\%). Other prescribed drugs were thalidomide (6.1\%), chlorambucil $(6.9 \%)$ methotrexate $(4.5 \%)$, anti-TNF-alpha therapies (infliximab $6.8 \%$ and adalimumab $2.3 \%)$, cyclophosphamide $(3 \%)$, mycophenolate $(3.8 \%)$, leflunomide $(1.5 \%)$ and $22 \%$ received anticoagulation.

Conclusion: Clinical features of our patients are similar to those of othe European cohorts, although a high prevalence of organic involvement (ocular, neurological, vascular and joint) should be highlighted.

\section{REFERENCES}

[1] Saadoun D, Wechsler B. Behçet's disease. Orphanet J Rare Dis. 2012 Apr 12;7;20. doi: 10.1186/1750-1172-7-20.

[2] Mendes D, et al. Behçet's disease - a contemporary rewiew. J Autoimmun. 2009 May-Jun; 32 (3-4): 178-88. doi: 10.1016/j.jaut.2009.02.011.

[3] Leccese $P$, et al. Behçet's síndrome in nonendemic regions. Curr Opin Rheumatol. 2017. doi: 10.1097/BOR.0000000000000349

Disclosure of Interests: None declared DOI: 10.1136/annrheumdis-2019-eular.6038

\section{AB0605 SERUM NEOPTERIN AND ISCHEMIA MODIFIED ALBUMIN LEVELS ARE ASSOCIATED WITH THE DISEASE ACTIVITY OF ADULT IMMUNOGLOBULIN A VASCULITIS (HENOCH-SCHONLEIN PURPURA)}

Ahmet Omma ${ }^{1}$, Seda Colak ${ }^{1}$, Sevinc Can Sandikci ${ }^{1}$, Cigdem Yucel $^{2}$, Abdulsamet Erden ${ }^{3}$, Erdim Sertoglu ${ }^{4}$, Taner Özgürtaş ${ }^{4} .{ }^{1}$ Health Sciences University, Ankara Numune Training and Research Hospital, Rheumatology, Ankara, Turkey; ${ }^{2}$ Health Sciences University, Ankara Numune Training and Research Hospital, Clinical Biochemistry, Ankara, Turkey, ${ }^{3}$ Yildirim Beyazit University, Rheumatology, Ankara, Turkey; ${ }^{4}$ University of Health Sciences, Gülhane School of Medicine, Medical Biochemistry, Ankara, Turkey

Background: Immunoglobulin A vasculitis (IgAV) [formerly called HenochSchönlein purpura (HSP)] is an $\lg A$ mediated systemic vasculitis which primarily affects skin, gastrointestinal system and small vessels of kid neys. Exact pathogenesis of IgAV remains unknown. A fewclinicalstudiesevaluatedthe role of oxidativeinjury in thepathogenesis of vasculitis. Ischemia modified albumine (IMA) and Neopterin increased status of oxidative stress.

Objectives: The aims of the study are to investigate serum neopterin and IMA levels in patients with IgAV and evaluate the association of these markers with disease activity and relapse.

Methods: Thirty-four consecutive adult patients (24 males and 10 females) admitted to the rheumatology clinic of Ankara Numune Training and Research Hospital meeting the IgAVAmerican College of Rheumatology (ACR) criteria were enrolled in this cross-sectional study. Demographic and clinical features of IgAV and control group were recorded into a predefined protocol. Disease activity was categorized as "remission" or "active" according to BVAS. BVAS $\geq 1$ was accepted "active".Serum neopterin levels, hsCRP and IMA were evaluated according to BVAS and compared to healthy control group.

Results: Serum median (IQR) neopterin, IMA levelsandhsCRPwerehigher in thestudygroupthan in controlgroup [2.01 (12.5) $\mathrm{ng} / \mathrm{mL}$ vs. 1.77 (1.37) $\mathrm{ng} / \mathrm{mL}, 0.67$ (0.2) ng/mL vs. $0.43(0.17) \mathrm{ng} / \mathrm{mL}, 5.6$ (17.1) mg/L vs. 1.55 (1.6) $\mathrm{mg} / \mathrm{L}, \quad \mathrm{p}=0.095, \quad \mathrm{p}<0.001$ and $\mathrm{p}=0.002$, respectively). When evaluated according to BVAS, IMA and hsCRP levels were significantly higher in the group with active disease $[0.77(0.12)$ vs. $0.61(0.13)$ and 14.85 (4.6), $p=0.009$ and $p=0.03$, respectively]. Serum neopterin levels were significantly higher in the active group compared to BVAS [18.95 (32.36) vs. 1.63 (1.48), $p<0.001]$.

Conclusion: Oxidative stress is important in HSP pathogenesis. Roles of hsCRP,Neopterin and IMA as potential markers of diagnosis and disease activity seem to be worth studying in the future studies with larger study groups

Disclosure of Interests: None declared

DOI: 10.1136/annrheumdis-2019-eular.3193 